\title{
Control de carga viral como estrategia de prevención. Indetectable=Intransmisible $(\mathrm{I}=\mathrm{I})$
}

Los efectos beneficiosos de la terapia antirretroviral (TARV) sobre la salud individual están bien establecidos. La TARV ha demostrado reducir la morbilidad y la mortalidad asociadas al VIH (1). Además, numerosos estudios observacionales sugirieron que el control de la carga viral a nivel poblacional se asociaba con una reducción en las nuevas infecciones en la comunidad $(2,3)$. Por ello, se considera que el tratamiento antirretroviral a nivel de la población ofrece tres tipos diferentes de impacto:

- Efecto individual, incluyendo mayor expectativa de vida (4), reducción del riesgo de sida y muerte por sida (5).

- Reducción de la transmisión secundaria $(6,7)$.

- Reducción en la tasa de infección por contacto $(8,9)$.

Sólidas evidencias soportan la no transmisibilidad del VIH en parejas serodiscordantes. En 2011 se conocieron los resultados del estudio HPTN 052, un ensayo controlado y aleatorizado que tuvo como objetivo evaluar el riesgo de transmisiones ligadas en parejas estables serodiscordantes, en el que fue encontrada una eficacia del tratamiento como prevención de 96\%; eso significa que las personas VIH positivas que tomaban ARV tuvieron 20 veces menos probabilidades de infectar a sus parejas que las personas no tratadas (10). El único caso de transmisión se produjo en una pareja en la que el hombre era el miembro $\mathrm{VIH}$ positivo y que tuvieron sexo no protegido pocos días luego del inicio del TARV. En el seguimiento a largo plazo la eficacia fue de $93 \%$, detectándose 8 infecciones en las parejas luego del inicio del TARV: 4 ocurrieron antes del inicio del TARV o inmediato al inicio del mismo y las otras 4 en el contexto de fallo virológico.

El estudio PARTNER 1 evaluó parejas serodiscordantes en las cuales el sujeto infectado tenía una determinación de carga viral menor a 200 copias $/ \mathrm{mm}^{3}$ y que reconocían haber tenido relaciones sexuales con penetración vaginal o anal sin preservativo en el mes previo (11). Fueron evaluadas 767 parejas que contribuyeron a 894 parejas/año de seguimiento para determinar infecciones por VIH filogenéticamente ligadas. Con una estimación de más de 44.000 relaciones registradas ( 16.400 relaciones entre HSH y 28.000 en parejas heterosexuales), no se produjo ningún caso de transmisión del VIH dentro de la pareja. Los resultados del estudio mostraron que el riesgo de transmisión más elevado marcado por el IC95\% fue de $0,45 \%$ anual en general, de 0,84\% para las relaciones sexuales entre $\mathrm{HSH}$ y de $4 \%$ en el caso del sexo anal receptivo con eyaculación.

El estudio PARTNERS 2 enroló parejas serodiscordantes de HSH dado el mayor límite superior del IC95\% en esta población, con las mismas características y metodología descriptas en PARTNERS 1 a partir de 2014 hasta abril de 2018 (12). Un total 
de 783 parejas contribuyeron a 1596 parejas/año de seguimiento, periodo durante el cual las parejas participantes mantuvieron aproximadamente 77.000 actos sexuales no protegidos. No se detectó ninguna infección por VIH filogenéticamente ligada, siendo el límite superior del IC95\% para el riesgo de trasmisión 0,23/100 y del 0,57\% en el caso del sexo anal receptivo con eyaculación. Estos IC95\% son considerablemente menores a los observados en PARTNERS 1.

Los resultados de PARTNERS 2 permiten afirmar que "indetectable es igual a intransmisible" para los HSH con igual nivel de certeza que para las parejas heterosexuales.

El estudio observacional Opposites Attract enroló parejas serodiscordantes de HSH en trece clínicas en Australia, una en Brasil y una en Tailandia (13). Fueron evaluables 343 parejas serodiscordantes en las cuales el sujeto infectado tuviera una carga viral menor a $200 \mathrm{copias} / \mathrm{ml}$. No se produjo ninguna infección por VIH ligada, siendo el límite superior del IC95\% de la tasa de transmisión 1,59 por cada 100 años de seguimiento.

Los estudios HPTN 052, PARTNERS 1 y 2, y Opposites Attract confirman la no transmisibilidad a nivel individual. Fue a partir de estos estudios que se ha generalizado el concepto de Indetectable=Intransmisible, I $=$ I o su traducción al inglés $\mathrm{U}=\mathrm{U}$. A la sólida evidencia que soporta el concepto de l=I se suma la ausencia de casos de transmisión ligada en individuos con replicación viral controlada.

El concepto I=I se refiere exclusivamente a la transmisión por vía sexual. No existen evidencias que permitan extrapolarlo a la transmisión por otras vías, como la lactancia o por el uso de jeringas u otros materiales cortopunzantes.

El tratamiento del VIH es una herramienta muy importante en la lucha contra el sida, pues previene enfermedades y muertes, evita nuevas infecciones y supone un ahorro económico. En una estrategia basada en derechos, los objetivos finales de acceso universal al tratamiento son esenciales, ya que las esperanzas de acabar con la epidemia de sida dependen en gran medida de la capacidad mundial de proporcionar tratamiento del VIH a todos aquellos que lo necesiten.

I=I, además de una poderosa herramienta para la prevención del VIH, es un mensaje que intenta desterrar la idea de la persona con VIH como "fuente de contagio".

\section{Gustavo Lopardo}

FUNCEI/Hospital Bernardo Houssay

Docente Enfermedades Infecciosas UBA 


\section{Referencias}

1. https://www.who.int/features/factfiles/hiv/es/

2. Das $M$, Chu PL, Santos GM, Scheer $S$, Scheer S, Vittinghoff E, McFarland W, et al. Decreases in community viral load are accompanied by reductions in new HIV infections in San Francisco. PLoS One. 2010; 5(6): e11068.

3. Montaner JS, Lima VD, Harrigan PR, Lourenço L, Yip B, Nosyk B et al 2014. Expansion of HAART coverage is associated with sustained decreases in HIV/AIDS morbidity, mortality and HIV transmission: the "HIV treatment as prevention" experience in a Canadian setting. PLoS ONE, 2014; 9: e87872.

4. Samji $\mathrm{H}$, Cescon A, Hogg RS, Modur SP, Althoff KN, Buchacz K, et al. Closing the gap: increases in life expectancy among treated HIV-positive individuals in the United States and Canada. PLoS One. 2013; 8: e81355.

5. Kitahata MM, Gange SJ, Abraham AG, Merriman B, Saag MS, Justice AC, et al. Effect of early versus deferredantiretroviral therapy for HIV on survival. $\mathrm{N}$ Engl $\mathrm{J}$ Med. 2009;360:1815-26.

6. Baeten JM, Donnell D, Ndase P, Mugo NR, Campbell JD, Wangisi J, et al. Antiretroviral prophylaxis for HIVprevention in heterosexual men and women. N Engl J Med. 2012; 367: 399-410.

7. Anglemyer A, Horvath T, Rutherford G. Antiretroviral therapy for prevention of HIV transmission in HIV-discordantcouples. JAMA. 2013; 310: 1619-20.

8. Rodger AJ, Cambiano V, Bruun T, Vernazza P, Collins S, Van Lunzen J, et al. Sexual activity without condoms and risk of HIV transmission in serodifferent couples when the HIV-positive partner is using suppressive antiretroviral therapy. JAMA. 2016; 316: 171-81.

9. Tanser F, Bärnighausen T, Grapsa E, Zaidi J, Newell ML. High coverage of ART associated with decline in risk of HIVacquisition in rural KwaZulu-Natal, South Africa. Science. 2013; 339: 966-71.

10. Cohen MS, Chen YQ, McCauley M, Gamble T, Hosseinipour MC, Kumarasamy N, et al. Antiretroviral Therapy for the Prevention of HIV-1 Transmission.NEngl J Med. 2016; 375(9): 830-9.

11. Rodger AJ, Cambiano V, Bruun T, Vernazza P, Collins S, van Lunzen J et al. Sexual Activity Without Condoms and Risk of HIV Transmission in Serodifferent Couples When the HIV-Positive Partner Is Using Suppressive Antiretroviral Therapy. JAMA. 2016; 316(2): 171-181.

12. A. Rodger, V. Cambiano, T. Bruun, P. Vernazza, S. Collins, G.M. Corbelli, et al. Risk of HIV transmission through condomless sex in MSM couples with suppressive ART: The PARTNER2 Study extended results in gay men. Program and abstracts of the 22nd International AIDS Conference; July 23-27, 2018; Amsterdam, the Netherlands. Abstract WEAX0104LB

13. Bavinton BR, Pinto AN, Phanuphak N, Grinsztejn B, Prestage GP, Zablotska-Manos IBet al. Viral suppression and HIV transmission in serodiscordant male couples: an international, prospective, observational, cohort study. Lancet HIV. 2018; 5(8): e438-e447. Epub 2018 Jul 17. Erratum in: Lancet HIV. 2018; 5(10): e545. 